\title{
Transfer inklusive
}

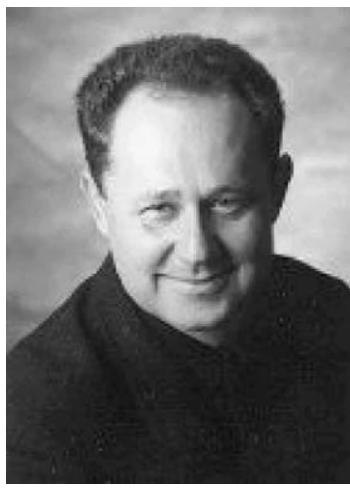

Prof. Dr. med. Manfred Wildner

\section{Bibliografie}

DOI http://dx.doi.org/ 10.1055/s-0032-1327646

Gesundheitswesen 2012;

74: 603-604

(c) Georg Thieme Verlag KG

Stuttgart · New York

ISSN 0941-3790

Korrespondenzadresse

Prof. Dr. med. Manfred Wildner

Bayerisches Landesamt für Gesundheit und Lebensmittelsicherheit

Veterinärstraße 2

85764 Oberschleißheim

manfred.wildner@|gl.bayern.de
Der Weg vom Ausgangsort zum Reiseziel kann mühsam und wenig motivierend sein. Der Pauschaltourismus hat darauf eine Antwort: Transfer inklusive. Die Reisenden werden auf ihrem Weg von zu Hause bis an ihr Ziel umsorgt: zu Lande, auf dem Wasser und in der Luft. Dies setzt ein erhebliches logistisches Know-how auf Seiten der Reiseveranstalter voraus. Der Kunde dankt dies mit seiner Buchung. Achtung: Die Preise müssen dabei so kalkuliert sein, dass dieser Transfer mit finanziert ist.

Was in der Reisebranche heute eine große Leichtigkeit erreicht hat, ist - im übertragenen Sinn im Bereich des Gesundheitswesens noch immer recht beschwerlich. Der Weg von der Idee bis zu ihrer Umsetzung ist häufig ein langer Marsch durch die Institutionen und Gremien, in denen die ursprünglichen Konzepte vielfältigen Änderungsanträgen und Kompromissen unterzogen werden. „Was ist ein Kamel? Ein Pferd, dessen Design in einem Komitee abgestimmt wurde“, so ein Bonmot.

Dieser lange Weg von der Idee bzw. der Erarbeitung von wissenschaftlicher Evidenz und theoriegestützten Konzepten bis in die praktische Umsetzung oder vorbereitend ihre Behandlung im politischen Umfeld wird zunehmend in seiner Bedeutsamkeit erkannt [1]. Unterschieden wird dabei eine Translationsphase 1 (T1) von den Grundlagenwissenschaften bis zur ersten Anwendung in Modellprojekten (,from bench to bedside“) und eine Translationsphase 2 (T2), dem Weg von Modellen guter Praxis zur Praxis guter Modelle. In letzterer geht es um die Umwandlung und Adaption von wissensbasierten Empfehlungen in praktische Maßnahmen (Knowledge Utilization), die damit verbundenen Aushandlungsprozesse zwischen den wissensbasierten Ausgangspunkten und den jeweiligen Settings ihrer Umsetzung (Implementation) und mehr allgemein um die aktiv gestaltete Verbreitung von Innovation (Dissemination) [2].

Auf Seiten der Wissenschaft und Forschung und des Wissenschaftsmanagements finden derartige Gedanken, gleichsam als Ausgang aus dem Elfenbeinturm, zunehmend Eingang. Beispiele geben diverse „Roadmaps“ des Forschungsmanagements großer Industrienationen $[3,4]$. Auch wenn der ursprüngliche Kristallisationspunkt dieser Anstrengungen im Bereich der Genomik und Proteomik liegt, sind im selben Sinn und in besonderer Weise zunehmend auch Gesundheitsförderung und Prävention anzusprechen. Noch allgemeiner betreffen diese Überlegungen den bevölkerungsbezogenen Health Impact von Epidemiologie und Public Health [5-8].
Dabei bewegt sich der Transfer bzw. die Translation von Wissen in die konkreten gesellschaftlichen Vollzüge in dem Dreiecksverhältnis von Wissenschaft und Praxis, von Wissenschaft und Politik und von Politik und Praxis [6]. Gerade im Verhältnis Wissenschaft und Praxis ist bisweilen schwer zu vermitteln, dass Evidenznutzung selten „all in one“ - wie durch den Zauberstab einer Fee oder die Transferleistungen im Pauschaltourismus - geschieht. Die Regel sind Stufen, die zu durchlaufen sind - bergauf, um im Bild zu bleiben. Rogers differenziert zwischen der Stufe 0, der klassischen Forschungsarbeit zur Evidenzgenerierung, der Stufe 1, der Dissemination der Evidenz und im Erfolgsfall der Stufe 2, der Adaption des Modells von der Zielgruppe sowie der Stufe 3, die Implementierung. Letztere wird ergänzt durch eine Stufe 4, der Institutionalisierung der entsprechenden Projektstrukturen und der Stufe 5, einer weiteren Verbreitung [2]. Dass diese Stufen nicht ohne Widerstände durchlaufen werden können, ist nahe liegend. Anreize und motivationale Aspekte sind daher von großer Bedeutung, Leitplanken und Barrieren sind zu unterscheiden.

Wie steht es um das Verhältnis von Wissenschaft und Politik? Um diese Gretchenfrage im Originalzitat zu stellen (Gretchen an Dr. Faustus): „Nun sag, wie hast du's mit der Religion? Du bist ein herzlich guter Mann, allein ich glaub du hältst nicht viel davon!" Zu diesem Nicht-viel-davonhalten des promovierten Kollegen Faust (mit gedanklichem Austausch von Religion durch Politik): Tatsächlich befinden sich an der Schnittstelle zur Politik Wissenschaftler subjektiv und objektiv in einer Sandwich-Position, eingepackt zwischen dem erkenntnisorientierten Werteparadigma der Wissenschaft und dem auf Machbarkeit, bisweilen auf Machterhalt ausgerichteten Paradigma der Politik. Gerade jüngere, gesinnungsethisch-idealistisch geprägte Wissenschaftler können von diesem Spannungsverhältnis überfordert werden, was dann gerne in eine generelle Anklage des „Politikbetriebs“ mündet. Hierbei werden Sie auch von erfahreneren, leise resignierenden Kollegen unterstützt, deren „Abgefrühstückt-werden“ an den grünen Tischen der Politik nun als Retourkutsche von einem überlegenen moralischen Niveau aus - dem wahrheits- und erkenntnisorientierten wissenschaftlichen Denk- und Handlungsrahmen - kritisiert wird.

Was leicht vergessen wird, ist die Unterschiedlichkeit des Handlungsrahmens auf der politischen Bühne mit seiner erheblichen Folgenschwere und Legitimationsbedürftigkeit jenseits der wissenschaftlichen Evidenz. Die ethische 
Grundposition der Politik ist verantwortungsethisch ausgerichtet, nicht gesinnungsethisch [9]. Politik befindet sich in ihren Entscheidungsfindungsprozessen in einem eigenen mehrstelligen Verhältnis. Dieses wird einerseits von der wissenschaftlichen Evidenz aufgespannt, andererseits auch von ethischen, juristischen und ökonomischen Imperativen - all das eingebettet in den kommunikativen Kontext einer Mediendemokratie mit ihren teilweise sehr kurzen Zeithorizonten. Dies mag vonseiten der Wissenschaft als kleines oder großes Staatstheater empfunden werden. „Reformulierung von Evidenzsynthesen als Rhetorik auf der Bühne des politischen Dramas“, raisonniert denn auch eine Arbeitsgruppe in Hinblick auf dieses Schauspiel [10]. Weiterführende Reflexionen geben das Memorandum Forschungsförderung Prävention [11] und eine Übersicht über Erfolge und Misserfolge der Gesundheitspolitik in Europa über einen Zeitraum von 4 Dekaden [12] - auf die breitere laufende wissenschaftliche Diskussion wird verwiesen [5,6,13-16].

Die Beiträge in diesem Heft bewegen sich allesamt zumindest in Teilen an dieser Schnittstelle von Wissenschaft, Politik und Praxis - ohne Anzeichen von Resignation erkennen zu lassen: Sie berichten zu Zukunftsthemen der Versorgungsforschung, zur Sichtweise der Kommunen auf den Hausärztemangel, zur Bedarfsgerechtigkeit der Bedarfsplanung, zu Unterschieden zwischen GKV- und PKV-Versicherten, zu den Kosten Osteoporosebedingter Frakturen, zur Prävention multiresistenter Erreger in der ambulanten Pflege und zur Validität von SprachscreeningInstrumenten in den pädiatrischen Vorsorgeuntersuchungen. Dazu tritt das Memorandum II zur Präventionsforschung sowie ein CME-zertifizierter Beitrag zum Schwerpunkt neues Entgeltsystem, Kodierrichtlinien in der Psychiatrie/Psychosomatik und ICD 10-GM/OPS.

Max Weber formulierte Anfang des 20. Jahrhunderts pointiert, dass Politik „ein starkes langsames Bohren von harten Brettern mit Leidenschaft und Augenmaß zugleich" bedeute [9; S. 66]. Beim Bohren der harten Bretter hilft wissenschaftlicher Rigor zweifelsohne, das ebenfalls angesprochene Augenmaß verbietet dabei ein Übermaß genauso wie ein Untermaß. Um an dieser Stelle das Eingangs vorgestellte Bild des Pferdes aufzugreifen, welches als Kamel eine Komitee-Sitzung wieder verlässt: Der
Transfer von Evidenz in die Praxis ist lang und steinig und kann von großer Trockenheit gekennzeichnet sein. So lange hierfür noch kein Pauschaltourismus, Transfer inklusive, angeboten wird, soll das langsamere Kamel auch einmal in Schutz genommen werden: Es könnte als Wüstentier vielleicht die bessere „evolutionäre“ Adaptation an die bevorstehenden Transferaufgaben darstellen als das ursprünglich vorgesehene Pferd.

\section{Literatur}

1 Green LW, Ottoson JM, Garciaq C et al. Diffussion Theory and Knowledge Dissemination, Utilization, and Integration in Public Health. Annual Rewiew of Public Health 2009; 30: 151-174

2 Rogers EM. Diffusion of Innovations. New York: Free Press; 2003

3 Zerhouni E. The NIG Roadmap. Science 2003; 302: 63-72

4 Gesundheitsforschungsrat des Bundesministeriums für Bildung und Forschung. Roadmap für das Gesundheitsforschungsprogramm der Bundesregierung. Bonn: GFR; 2007

5 Rütten A, Gelios P. Evidenzbasierte Politik und nachhaltiger Wissenstransfer: Eine Perspektive für die Gesundheitsförderung in Deutschland. Gesundheitswesen 2012; 4: 224-228

6 Wildner M. Prävention an den Schnittstellen zur Politik und Praxis. Gesundheitswesen 2012; 4: 229-233

7 Frieden TR. A framework for public health action: the health impact pyramid. Am J Public Health 2010; 100: 590-595

8 Khoury MJ, Gwinn M, Ioannidis JP. The emergence of translational epidemiology: from scientific discovery to population health impact. Am J Epidemiol 2010; 172: 517-524

9 Weber M. Politik als Beruf. München und Leipzig: Duncker und Humblot 1919 (Nachdruck: Stuttgart: Reclam; 1992)

10 Greenhalgh T, Russell J. Reframing Evidence Synthesis as Rhetorical Action in the Policy Making Drama. Healthcare Policy 2005; 1: 31-39

11 Walter $U$, Gold C, Hoffmann $W$ et al. Memorandum - Forschungsförderung Prävention. Gesundheitswesen 2012; 74: 526-532

12 McKee M, Mackenbach J, Hrsg. Successes and failures of health policy in Europe over four decades. (Im Druck)

13 Knieps F. Evidence based health policy oder wissenschaftlich verbrämter Lobbyismus - die Verwertung wissenschaftlicher Erkenntnisse in der Gesundheitspolitik. Z f Evidenz Fortb Qualität Gesundheitswesen 2009; 103: 273-380

14 Haynes AS, Derrick GE, Chapman S et al. From "our world" to the "real world": exploring the views and behaviour of policy-influential Australian public health researchers. Soc Sci Med 2011; 72: 1047-1055

15 Lavis JN, Posada FB, Haines $A$ et al. Use of research to inform public policymaking. Lancet 2004; 364: 1615-1621

16 Weltgesundheitsorganisation (WHO). Bridging the „Know-Do“ Gap. Meeting on Knowledge Translation in Global Health. Genf: WHO; 2005 